\title{
Identification of ABCA1 and ABCG1 in milk fat globules and mammary cells-Implications for milk cholesterol secretion
}

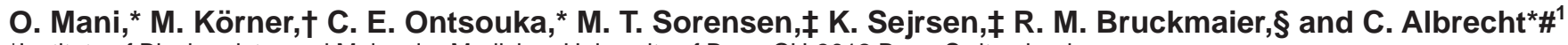 \\ *Institute of Biochemistry and Molecular Medicine, University of Bern, CH-3012 Bern, Switzerland \\ †Institute of Pathology, University of Bern, $\mathrm{CH}-3010$ Bern, Switzerland \\ ‡Department of Animal Health, Welfare and Nutrition, Aarhus University, DK-8830 Tjele, Denmark \\ §Veterinary Physiology, Vetsuisse Faculty, University of Bern, CH-3001 Bern, Switzerland \\ \#Swiss National Center of Competence in Research, NCCR-TransCure, University of Bern, $\mathrm{CH}-3012$ Bern, Switzerland
}

\section{ABSTRACT}

The ATP-binding cassette (ABC) transporters ABCA1 and ABCG1 play an important role in cellular cholesterol homeostasis, but their function in mammary gland (MG) tissue remains elusive. A bovine MG model that allows repeated MG sampling in identical animals at different functional stages was used to test whether 1) ABCA1 and ABCG1 protein expression and subcellular localization in mammary epithelial cells (MEC) change during the pregnancy-lactation cycle, and 2) these 2 proteins were present in milk fat globules (MFG). Expression and localization in MEC were investigated in bovine MG tissues at the end of lactation, during the dry period (DP), and early lactation using immunohistochemical and immunofluorescence approaches. The presence of ABCA1 and ABCG1 in MFG isolated from fresh milk was determined by immunofluorescence. The ABCA1 protein expression in MEC, expressed as arbitrary units, was higher during the end of lactation (12.2 $\pm 0.24)$ and the DP $(12.5 \pm 0.22)$ as compared with during early lactation $(10.2 \pm 0.65)$. In contrast, no significant change in ABCG1 expression existed between the stages. Throughout the cycle, ABCA1 and ABCG1 were detected in the apical $(41.9 \pm 24.8$ and $49.0 \pm$ $4.96 \%$ of cows, respectively), basal (56.2 \pm 28.1 and $54.6 \pm 7.78 \%$ of cows, respectively), or entire cytoplasm $(56.8 \pm 13.4$ and $61.6 \pm 14.4 \%$ of cows, respectively) of MEC, or showed combined localization. Unlike ABCG1, ABCA1 was absent at the apical aspect of MEC during early lactation. Immunolabeling experiments revealed the presence of $\mathrm{ABCA} 1$ and $\mathrm{ABCG} 1$ in MFG membranes. Findings suggest a differential, functional stage-dependent role of ABCA1 and ABCG1 in cholesterol homeostasis of the MG epithelium. The presence of ABCA1 and ABCG1 in MFG membranes

Received June 9, 2010

Accepted November 1, 2010.

${ }^{1}$ Corresponding author: christiane.albrecht@mci.unibe.ch suggests that these proteins are involved in cholesterol exchange between MEC and alveolar milk.

Key words: ABC transporter, cholesterol homeostasis, mammary gland, milk fat globule

\section{INTRODUCTION}

With the onset of lactation, mammary epithelial cells (MEC) differentiate into a lactating epithelium. Lipids, including cholesterol, are not only important membrane components but also milk nutrients needed for neonatal development. The transport and distribution of cholesterol in MEC is vital for cellular functions as well as for further release into milk. The uptake of cholesterol from the blood into peripheral cells and the cellular redistribution includes interactions of lipid carriers and lipid receptors, followed by the active or passive uptake processes into the cell, and redistribution to other cellular compartments by different accessory proteins (Ioannou, 2001; Ikonen, 2008).

The ATP-binding cassette (ABC) transporters ABCA1 and ABCG1 are integral membrane proteins that, in peripheral cells, mediate an active efflux of lipophilic substrates such as phospholipids and cholesterol to lipid-poor apolipoprotein (apo) A1 and mature high-density lipoprotein, respectively, through the hydrolysis of ATP (Takahashi et al., 2005; Cavelier et al., 2006). As such, these proteins may play a role in mammary gland (MG) physiology and may be involved in milk cholesterol release.

Previous investigations in dairy cows revealed that mRNA transcripts of $\mathrm{ABC}$ transporters, including ABCA1 and ABCG1, exhibit differential expression patterns and are inversely correlated to circulating cholesterol during the pregnancy-lactation cycle (Mani et al., 2009). In vitro investigations using HeLa cells showed the presence of ABCA1 protein on the cell surface as well as on intracellular vesicles, suggesting a possible trafficking of the protein between intracellular compartments and the cell surface (Neufeld et al., 2001). On the other hand, the presence of ABCA1 
protein in cytoplasmic structures of bovine MEC with apical and basal accentuation was reported using immunohistochemistry (Mani et al., 2009). Milk fat globules (MFG) are a key constituent of the secretory pathway in MEC and are composed of a triglyceride-rich core which harbors a specific composition. They are surrounded by an inner monolayer of amphiphilic lipids and proteins, and an outer lipid bilayer derived from the apical part of MEC (McManaman and Neville, 2003), termed the outer MFG membrane (MFGM). As demonstrated in rats, cholesterol of dietary origin is believed to be transferred to the apical membrane of MEC and to be incorporated into the MFGM (Easter, 1971). Studies performed in cows reported a variation in cholesterol and triglyceride contents of MFGM during the pregnancy-lactation cycle (Bitman et al., 1992) as well as changes in the expression of specific proteins (e.g., apo A1), which may act as cholesterol acceptors (Reinhardt and Lippolis, 2008). Furthermore, ABCG2, also known as breast cancer resistance protein, which is structurally and functionally related to ABCA1 and ABCG1, was identified at the apical side of MEC among various species including Bos taurus (Robenek et al., 2006; Fong et al., 2007; Reinhardt and Lippolis, 2008) as well as in the MFGM (Fortunato et al., 2003; Fong et al., 2007).

The present study was undertaken to evaluate the protein abundance, cellular distribution, and subcellular localization of the cholesterol transporters ABCA1 and ABCG1 in MG tissues of lactating and nonlactating dairy cows. We tested the hypotheses 1 ) that ABCA1 and ABCG1 are present in MFG isolated from mature fresh milk of lactating dairy cows, and 2) that ABCA1 and ABCG1 show a differential protein expression and subcellular localization pattern during the pregnancylactation cycle.

\section{MATERIALS AND METHODS}

\section{Animals, Diets, and Sampling}

The animal experimentation was conducted according to the guidelines of the Danish Ministry of Justice on Animal Experimentation and Care. The protocols were approved by the Danish Animal Experiments Inspectorate. The experimental setup, animal characteristics, and sampling procedures were as described previously (Sorensen et al., 2006). Briefly, Holstein-Friesian dairy cows were housed in tie-stalls. They were milked twice daily and fed a ration based on grass silage and concentrate. The feeding regimen was ad libitum throughout lactation and restricted during the DP according to current cattle feeding recommendations. Pregnant cows were dried off $52 \mathrm{~d}$ before the next expected parturition (d -52). The MG biopsies were taken from the front quarter of each cow at the end of the previous lactation ( $d-77$ before the next parturition, $\mathrm{n}=7$ ), during the DP (d $-16, \mathrm{n}=5)$, and on d 42 after parturition ( $=6$ ). The collected biopsies were fixed overnight in $4 \%$ neutral buffered formalin before being dehydrated and embedded in paraffin. The milk yield of cows was within the expected range and was not affected by biopsy sampling.

Because the milk from biopsied animals was lacking, fresh raw milk collected from dairy cows of an organic farm (Hinterkappelen, Bern, Switzerland) was used for MFG preparation. The bulk tank milk used for the experiment was derived from 18 cows, which represented different stages of lactation from wk 2 to 40. Lactation numbers of the cows ranged from 1 to 6 . The SCC of the milk was around $190,000 / \mathrm{mL}$. Thus, the milk quality was within the standards of the Swiss Consumer Health Association, which requires a SCC $<350,000 /$ $\mathrm{mL}$ for consumed milk.

\section{Tissue Immunohistochemistry and Immunofluorescence}

Serial sections of paraffin-embedded tissue $(4 \mu \mathrm{m})$ were prepared and picked up on SuperFrost slides (SuperFrost, Braunschweig, Germany). After drying, sections were dewaxed in xylol and rehydrated through graded alcohols. Endogenous peroxidase activity was blocked by incubating the slides in $3 \% \mathrm{H}_{2} \mathrm{O}_{2}$ solution for $10 \mathrm{~min}$ at room temperature (RT). An antigen recovery step in sodium citrate buffer $(10 \mathrm{~m} M, \mathrm{pH} 5.5)$ was performed in a microwave oven at $98^{\circ} \mathrm{C}$ for $6 \mathrm{~min}$ at $450 \mathrm{~W}$. After blocking unspecific binding with $4 \%$ of BSA for $1 \mathrm{~h}$ at RT, slides were incubated in a humid chamber at $4^{\circ} \mathrm{C}$ overnight with or without (negative controls) specific antibodies against human ABCA1 (Abcam, ab 7360, Cambridge UK; polyclonal) and human ABCG1 (LS-B507, LifeSpan BioSciences, Seattle, WA; polyclonal). Cross-reactivity of both antibodies in bovine mammary tissue was previously shown (Mani et al., 2009, 2010). Specificity of the antibodies was verified by immunoblotting (ABCA1 and ABCG1) and competitive immunoinhibition (only ABCA1; Paul et al., 2008). The use of these antibodies in immunohistochemistry was validated across a panel of formalin-fixed, paraffin-embedded tissues. In this context the stained slides were evaluated by a pathologist to confirm staining specificity. Both antibodies were applied at a concentration of $10 \mu \mathrm{g} / \mathrm{mL}$. After washing the slides, affinity-purified and biotin-conjugated polyclonal goat anti-rabbit IgG purchased from Dako (cat. no. E0432, Glostrup, Denmark) in a dilution of 5 $\mu \mathrm{g} / \mathrm{mL}$ was applied for $60 \mathrm{~min}$ at $\mathrm{RT}$ in a humidified 
chamber. The slides were washed and then exposed for $45 \mathrm{~min}$ to avidin-biotin-horseradish peroxidase complex prepared from reagents supplied by Dako (cat. no. K0377). Bound peroxidase was detected following 7 to 12 min incubation at RT with 3,3'-diaminobenzidine substrate. Finally, sections were briefly counterstained with hematoxylin and mounted in Aquatex (Merck, Darmstadt, Germany).

The detection of labeled ABCA1 and ABCG1 by immunofluorescence was performed as described above with the following modifications: (1) both primary antibodies were applied at a concentration of $1 \mu \mathrm{g} / \mathrm{mL}$, (2) the blocking step with $\mathrm{H}_{2} \mathrm{O}_{2}$ was omitted, and (3) Alexa Fluor 488 conjugated goat anti-rabbit polyclonal secondary antibodies (Invitrogen AG, Basel, Switzerland) were applied at a concentration of $0.5 \mu \mathrm{g} / \mathrm{mL}$ for $1 \mathrm{~h}$. In all experiments, negative controls consisted of both IgG affinity purified isotype samples from the host serum (Sigma-Aldrich, Basel, Switzerland) and secondary antibodies alone.

\section{Isolation and Fixation of MFG for Immune Labeling}

Two 15-mL falcon tubes filled with fresh raw cow milk were centrifuged at $450 \times g$ for 12 min at $4^{\circ} \mathrm{C}$. A 1-mL aliquot of the supernatant (cream layer) was added to $9 \mathrm{~mL}$ of chilled 0.1 $M$ PBS (pH 7.4) and the solution was accurately mixed by inversion. A $1-\mathrm{mL}$ aliquot of this suspension was mixed with $9 \mathrm{~mL}$ of $4 \%$ paraformaldehyde (PFA) on ice. Finally, $150 \mu \mathrm{L}$ of PFA-suspended cream was pipetted onto uncoated plastic chamber slides (Nunc, Thermo Fisher Scientific, Langenselbold, Germany) to allow fixation of the MFG to the bottom of the chamber slides. After $4 \mathrm{~h}$ incubation at RT, excess fluid was removed. The remaining MFG were dried overnight at RT.

\section{Immunochemistry of MFG}

Isolated and PFA-fixed MFG on chamber slides were used for the detection of ABCA1 and ABCG1, triglycerides, and cholesterol. Dried and fixed MFG were washed 3 times in PBS and then permeabilized in PBS containing $0.25 \%$ of Triton X-100 and $0.05 \%$ of Tween-20 for $10 \mathrm{~min}$ at RT. Permeabilized MFG were washed twice with PBS to remove remaining detergents. Prior to immunolabeling, the permeabilized MFG were incubated with blocking solution (PBS containing $1 \%$ of BSA, $2 \%$ of goat serum, and $5 \%$ of dried skim milk) for $1 \mathrm{~h}$. In order to detect ABCA1 and ABCG1, the specific rabbit polyclonal antibodies against bovine ABCA1 and ABCG1 (see above) were diluted in blocking solution and incubated overnight at $4^{\circ} \mathrm{C}$ in a humidified chamber at a concentration of
$2 \mu \mathrm{g} / \mathrm{mL}$. The primary antibody-labeled ABCA1 and ABCG1 proteins were detected with an Alexa Fluor 488 conjugated goat anti-rabbit IgG polyclonal antibody (Invitrogen) diluted in 1\% of BSA and $0.05 \%$ of Tween-20 in PBS (final concentration $0.5 \mu \mathrm{g} / \mathrm{mL}$ ).

Triglycerides in the MFG core were stained with $0.2 \%$ (wt/vol) Oil Red O dye (ORO; Sigma). Prior to staining, the samples were equilibrated in $60 \%$ isopropanol for $5 \mathrm{~min}$. The ORO solution $(0.35 \% \mathrm{wt} / \mathrm{vol})$ was filtered through a $0.2-\mu \mathrm{m}$ glass fiber filter (Semadeni, Ostermundigen, Switzerland) and then diluted 1:2 with isopropanol. The MFG were incubated with this solution for 30 min at RT, then rinsed with $60 \%$ isopropanol and rehydrated with distilled water.

In order to detect cholesterol in the MFGM, MFG were fixed on chamber slides as described above and were incubated with filipin complex (Sigma; $0.5 \mathrm{mg}$ / $\mathrm{mL}$ diluted in PBS containing 1\% of DMSO) for $1 \mathrm{~h}$ at RT in the dark. All specimens were mounted with fluorescent mounting medium (Dako).

\section{Microscopy}

The 3,3'-diaminobenzidine substrate-labeled sections were visualized with a bright field microscope (Leica Microsystems GmbH, Wetzlar, Germany) using a $20 \times$ objective. Fluorescent dyes were visualized by using an Eclipse C1 laser confocal scanning microscope system (Nikon). Alexa Fluor 488 fluorescent dye was excited by an argon-ion laser (CVI Melles-Griot, Bensheim, Germany) at $488 \mathrm{~nm}$; ORO lipid stain was excited with a He-Ne laser (JDS Uniphase, Milpitas, CA) at 594 $\mathrm{nm}$. The 4'-6-diamidino-2-phenylindole (DAPI) bound to DNA was excited with a diode laser (Melles-Griot) at $405 \mathrm{~nm}$ and detected at $447 \mathrm{~nm}$. For transmission, specimens were irradiated with the $488 \mathrm{~nm}$ laser. Emitted fluorescence was detected at $525 \mathrm{~nm}$ (green) and $628 \mathrm{~nm}$ (red) by an Eclipse TE2000 inverted microscope C1 scanning head (Nikon). Filipin bound to cholesterol was visualized on a fluorescence microscope (Leica) using a $100 \times$ objective and a Hoechst filter (340 to 380 $\mathrm{nm}$ excitation, detection at $430 \mathrm{~nm})$. Confocal image processing was performed using the EZ-C1 FreeViewer (Version 3.90) software (Nikon Instruments Europe B.V., Amstelveen, the Netherlands). Bright field and fluorescence microscope images were processed using Adobe Photoshop software.

\section{Semiquantitative Evaluation of ABCA1 and ABCG1 Protein}

A pathology expert who was unaware of the experimental design estimated the percentage as well as the intensity of MEC staining for ABCA1 and ABCG1 for 
Table 1. Subcellular localization and semiquantitative evaluation of the ATP-binding cassette transporter A1 (ABCA1) protein expression in mammary epithelial cells (MEC) of individual animals during the pregnancy-lactation cycle ${ }^{1}$

\begin{tabular}{|c|c|c|c|c|c|}
\hline $\begin{array}{l}\text { Day relative to } \\
\text { parturition }\end{array}$ & $\begin{array}{l}\text { Animal } \\
\text { number }\end{array}$ & $\begin{array}{l}\text { Subcellular } \\
\text { localization }^{2}\end{array}$ & $\begin{array}{l}\text { Staining } \\
\text { intensity }\end{array}$ & $\begin{array}{c}\% \text { of epithelia } \\
\text { stained }\end{array}$ & $\begin{array}{l}\text { Protein } \\
\text { expression } \\
\text { grade }\end{array}$ \\
\hline \multirow[t]{4}{*}{$\mathrm{d}-77$ (late lactation) } & 1 & $a, b$ & + & $>90$ & 11.0 \\
\hline & 3 & $a, b$ & ++ & $>90$ & 12.0 \\
\hline & 4 & $a, b, e$ & ++ to +++ & $>90$ & 12.5 \\
\hline & 5 & $a, b$ & ++ to +++ & $>90$ & 12.5 \\
\hline \multirow[t]{5}{*}{ d -16 (dry period, late pregnancy) } & 1 & a & +++ & $>90$ & 13.0 \\
\hline & 2 & $\mathrm{a}, \mathrm{e}$ & ++ & $>90$ & 12.0 \\
\hline & 3 & e & ++ to +++ & $>90$ & 12.5 \\
\hline & 5 & $\mathrm{e}$ & ++ & $>90$ & 12.0 \\
\hline & 7 & $\mathrm{e}, \mathrm{m}$ & +++ & $>90$ & 13.0 \\
\hline \multirow[t]{2}{*}{ d 42 (early lactation) } & 1 & $\mathrm{e}, \mathrm{b}, \mathrm{m}$ & ++ & $>90$ & 12.0 \\
\hline & 2 & $\mathrm{~m}$ & ++ & 50 to 60 & 8.0 \\
\hline
\end{tabular}

${ }^{1}$ The procedure used for determination of the protein expression grade was as follows: first, the estimated percentage of MEC staining was transformed to grading scores $(\mathrm{n})$ using the following rule: $\%$ of positive cells $\leq 10 \% \rightarrow \mathrm{n}=1,11$ to $20 \% \rightarrow \mathrm{n}=2,21$ to $30 \% \rightarrow \mathrm{n}=3,31$ to $40 \% \rightarrow$ $\mathrm{n}=4,41$ to $50 \% \rightarrow \mathrm{n}=5,51$ to $60 \% \rightarrow \mathrm{n}=6,61$ to $70 \% \rightarrow \mathrm{n}=7,71$ to $80 \% \rightarrow \mathrm{n}=8,81$ to $90 \% \rightarrow \mathrm{n}=9$, and $>90 \% \rightarrow \mathrm{n}=10$. Then, the intensity of the staining, which varied from low to high, was estimated in a range from 1 to $3(+=1,+$ to $++=1.5,++=2,++$ to $+++=$ 2.5 , and $+++=3$ ) and was added to the grading scores to obtain the final protein expression grade in each cow.

${ }^{2}$ The subcellular localization patterns of ABCA1 in MEC of lactating ( $\mathrm{d}-77$ before parturition; $\mathrm{d} 42$ after parturition) and nonlactating dairy cows (d -16, dry period) were evaluated using immunohistochemically stained sections, as shown in Figure 1. a = staining of apical cytoplasm, $\mathrm{b}=$ staining of basal cytoplasm, $\mathrm{e}=$ staining of entire cytoplasm, $\mathrm{m}=$ membrane staining.

each section and animal, and defined the subcellular localization of both proteins. A frequently used scoring system (Paterson et al., 1990; Mohsin et al., 2004) was adapted by transforming the estimated percentage of MEC staining for ABCA1 and ABCG1 into a protein expression grade. In addition, a correction factor for staining intensity was included. First, the estimated percentage of MEC staining was transformed to grading scores (n) using the following rule: $\%$ of positive cells $\leq 10 \% \rightarrow \mathrm{n}=1,11$ to $20 \% \rightarrow \mathrm{n}=2,21$ to $30 \%$ $\rightarrow \mathrm{n}=3,31$ to $40 \% \rightarrow \mathrm{n}=4,41$ to $50 \% \rightarrow \mathrm{n}=5,51$ to $60 \% \rightarrow \mathrm{n}=6,61$ to $70 \% \rightarrow \mathrm{n}=7,71$ to $80 \% \rightarrow \mathrm{n}$ $=8,81$ to $90 \% \rightarrow \mathrm{n}=9$, and $>90 \% \rightarrow \mathrm{n}=10$. Then, the intensity of the staining, which varied from low to high, was estimated in a range from 1 to $3(+=1,+$ to $++=1.5,++=2,++$ to $+++=2.5$, and $+++=3)$ and was added to the grading scores to obtain the final protein expression grade in each cow (Tables 1 and 2).

\section{Statistical Evaluations}

For evaluation of the results, the statistical software SigmaStat 3.5 was used (Systat Software Inc., San Jose, CA). To identify differences between the investigated stages of the pregnancy-lactation cycle $(\mathrm{d}-77, \mathrm{~d}-16$, and $\mathrm{d} 42$ ), protein expression grades of ABCA1 and ABCG1 were evaluated by one-way ANOVA for repeated measures. The model was as follows:

$$
\begin{aligned}
& Y_{i j k}=\mu+A B C_{i}+\text { stage } P_{j} \\
& +(A B C \times \text { stage } P L)_{i j}+e_{i j k},
\end{aligned}
$$

where $Y_{i j k}$ represented the measured value, $\mu$ represented the general mean, $A B C$ represented the transporter types, stage $P L$ represented the stages of the pregnancylactation cycle, and $e_{i j k}$ represented the residual error. The $A B C$ and stage $P L$ were used as fixed effects and the individual cows were used as random effects. Correction according to Bonferroni was used to adjust for repeated testing. The level of significance was set at $P$ $<0.05$.

Table 2. Semiquantitative analysis of the ATP-binding cassette transporters A1 (ABCA1) and G1 (ABCG1) protein expression in mammary epithelial cells (MEC) in lactating and nonlactating Holstein dairy cows ${ }^{1}$

\begin{tabular}{lcr}
\hline $\begin{array}{l}\text { Functional } \\
\text { stage }\end{array}$ & ABCA1 & \multicolumn{1}{c}{ ABCG1 } \\
\hline $\mathrm{d}-77$ & $12.20 \pm 0.24^{\mathrm{a}}$ & $7.78 \pm 1.85$ \\
$\mathrm{~d}-16$ & $12.50 \pm 0.22^{\mathrm{a}}$ & $10.50 \pm 1.16$ \\
$\mathrm{~d} 42$ & $10.20 \pm 0.65^{\mathrm{b}}$ & $8.67 \pm 1.65$ \\
\hline
\end{tabular}

${ }^{\mathrm{a}, \mathrm{b}}$ Means with different superscripts within the column are different $(P<0.05)$.

${ }^{1}$ Indicated values (means \pm SEM) are based on Tables 1 and 3 and are expressed as arbitrary units. 
A
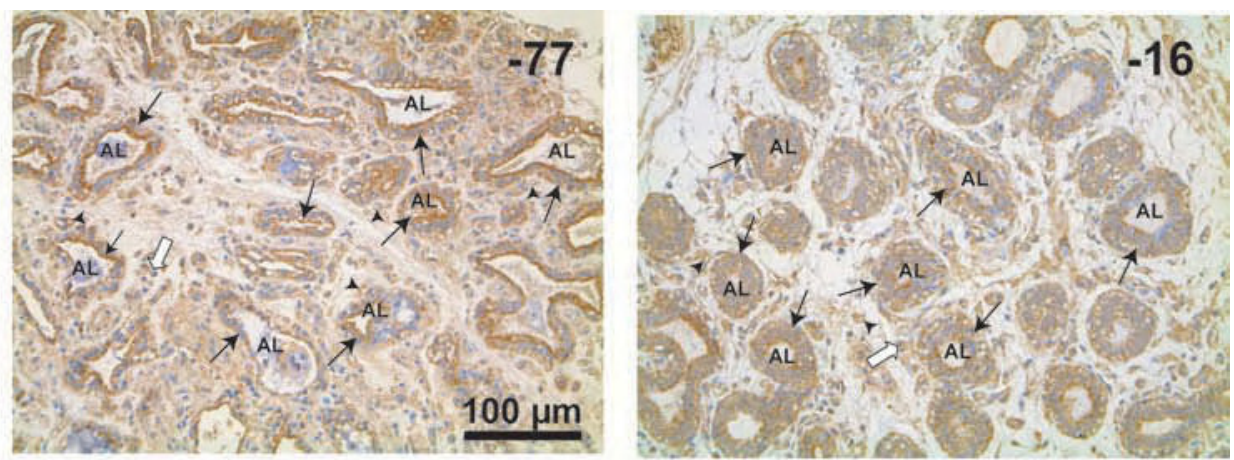

ABCA1
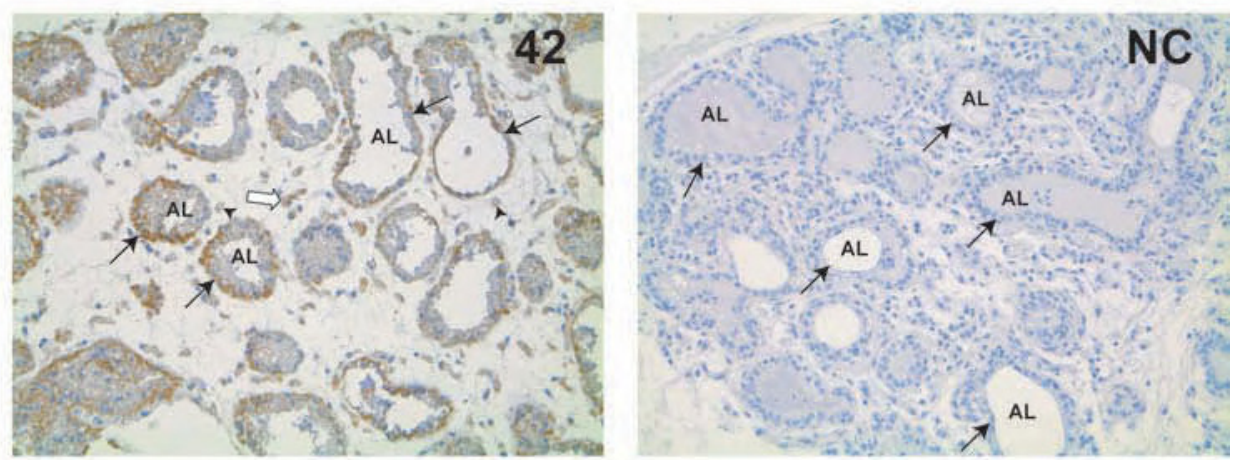

B
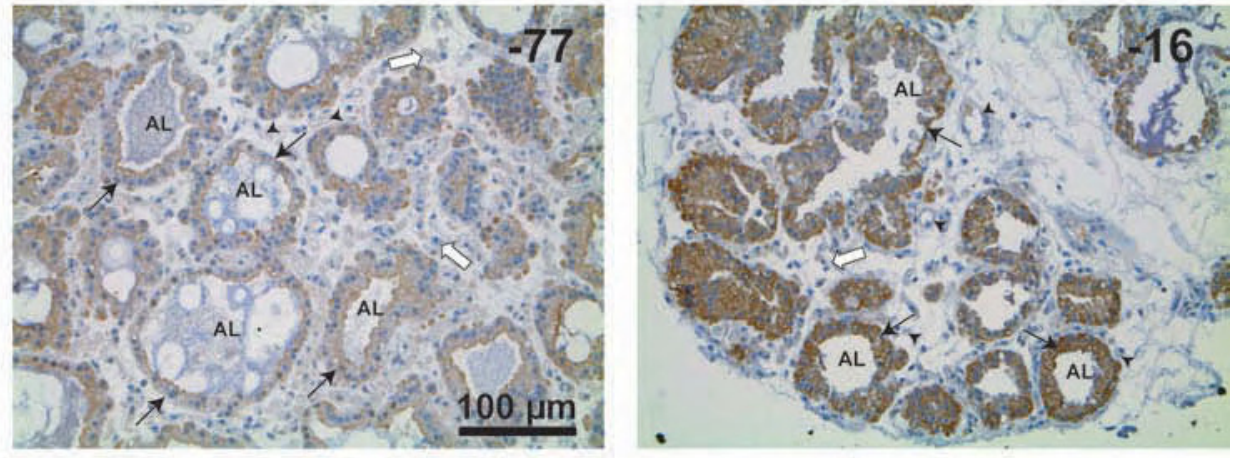

ABCG1
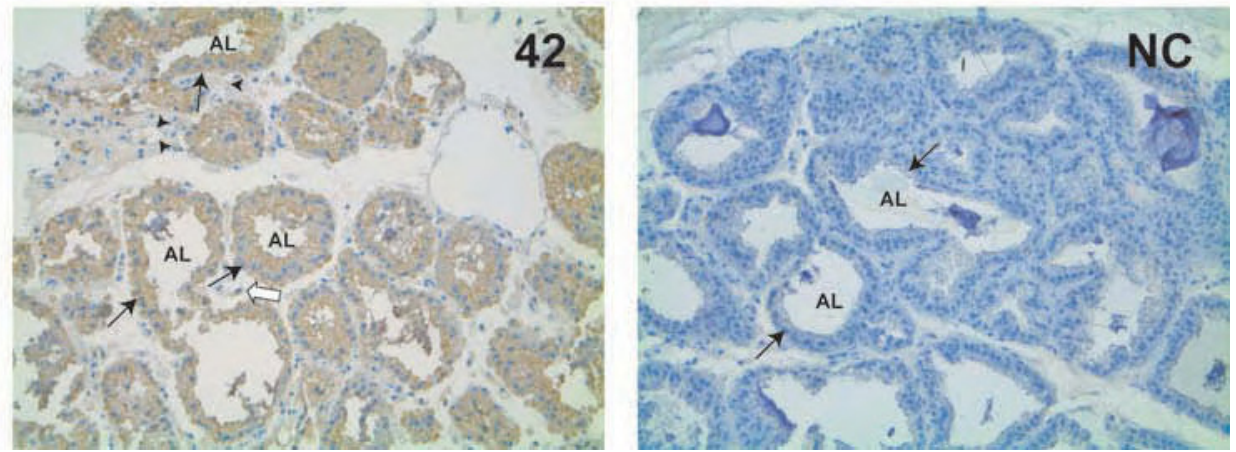

Figure 1. Distribution of the ATP-binding cassette transporters A1 (ABCA1) and G1 (ABCG1) in mammary tissues of lactating and nonlactating dairy cows. The images illustrate the subcellular localization and expression grade of ABCA1 (panel A) and ABCG1 (panel B) at the end of the previous lactation ( $\mathrm{d}-77$ to parturition), dry period (d -16 to parturition), and early lactation (d 42 after parturition). Bright field pictures were recorded on an inverted microscope using a color camera and a $20 \times$ objective. The method used for the evaluation of the expression grade is described in materials and methods. The NC are negative controls using only secondary antibody. Black arrows indicate the mammary epithelium that forms the alveolar lumen (AL). Between the alveoli, stromal mesenchymal cells (white arrows) and blood vessels (black arrowheads) are present in the stroma. Color version available in the online PDF. 


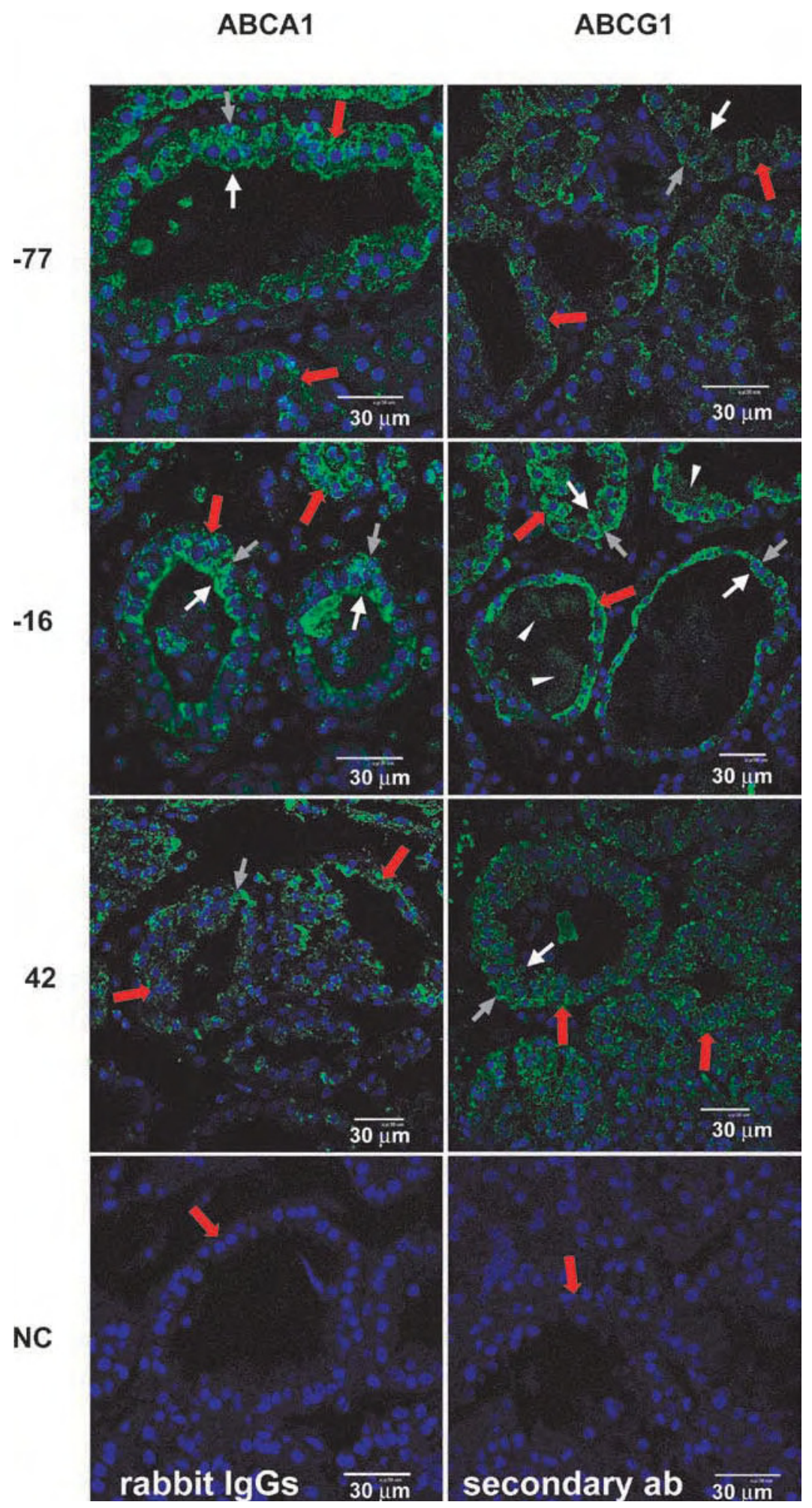

Figure 2. Subcellular localization of the ATP-binding cassette transporters A1 (ABCA1) and G1 (ABCG1) in mammary tissues of lactating and nonlactating dairy cows using immunofluorescence. Sections from paraffin-embedded biopsies stained for ABCA1 (left column) and ABCG1 (right column) were collected from the same animals at the end of the previous lactation ( $\mathrm{d}-77$ to parturition), dry period (d -16 to parturition), and early lactation (d 42 after parturition). Nuclei were counterstained with 4'-6-diamidino-2-phenylindole (DAPI; blue). Negative controls $(\mathrm{NC})$ are shown in the lower panel. Images were recorded by laser scanning confocal microscopy using a $40 \times$ objective. Red arrows indicate mammary epithelial cells (MEC). ABCA1 (left panels) or ABCG1 (right panels) show apical (white arrows) or basal (gray arrows) localization in MEC; white arrowheads indicate ABCG1 in intraluminal secretions (d - 16; right panel). Color version available in the online PDF. 
Table 3. Subcellular localization and semiquantitative evaluation of the ATP-binding cassette transporter G1 (ABCG1) protein expression in mammary epithelial cells (MEC) of individual animals during the pregnancy-lactation cycle ${ }^{1}$

\begin{tabular}{|c|c|c|c|c|c|}
\hline $\begin{array}{l}\text { Day relative to } \\
\text { parturition }\end{array}$ & $\begin{array}{l}\text { Animal } \\
\text { number }\end{array}$ & $\begin{array}{l}\text { Subcellular } \\
\text { localization }^{2}\end{array}$ & $\begin{array}{l}\text { Staining } \\
\text { intensity }\end{array}$ & $\begin{array}{c}\% \text { of epithelia } \\
\text { stained }\end{array}$ & $\begin{array}{l}\text { Protein } \\
\text { expression } \\
\text { grade }\end{array}$ \\
\hline \multirow[t]{4}{*}{$\mathrm{d}-77$ (late lactation) } & 1 & $\mathrm{a}, \mathrm{b}, \mathrm{e}$ & ++ & 20 & 4.0 \\
\hline & 3 & $a, b$ & + & $<10$ & 2.0 \\
\hline & 4 & $\mathrm{a}, \mathrm{b}, \mathrm{e}, \mathrm{m}$ & + to ++ & 80 to 90 & 10.5 \\
\hline & 5 & $\mathrm{~b}, \mathrm{e}, \mathrm{m}$ & ++ & 80 to 90 & 11.0 \\
\hline \multirow[t]{5}{*}{$\mathrm{d}-16$ (dry period, late pregnancy) } & 1 & $\mathrm{a}, \mathrm{b}, \mathrm{m}$ & + to ++ & 80 & 10.5 \\
\hline & 2 & $\mathrm{e}, \mathrm{m}$ & ++ & $>90$ & 12.0 \\
\hline & 3 & $\mathrm{e}$ & + & 40 to 50 & 6.0 \\
\hline & 5 & $\mathrm{e}, \mathrm{m}$ & ++ & $>90$ & 12.0 \\
\hline & 7 & $\mathrm{a}, \mathrm{b}, \mathrm{e}$ & +++ & 80 to 90 & 12.0 \\
\hline \multirow{2}{*}{ d 42 (early lactation) } & 1 & $\mathrm{e}$ & + to ++ & 60 to 70 & 8.5 \\
\hline & 2 & $\mathrm{a}, \mathrm{b}$ & + & 40 to 50 & 6.0 \\
\hline
\end{tabular}

${ }^{1}$ The procedure used for determination of the protein expression grade was as follows: first, the estimated percentage of MEC staining was transformed to grading scores ( $\mathrm{n}$ ) using the following rule: $\%$ of positive cells $\leq 10 \% \rightarrow \mathrm{n}=1,11$ to $20 \% \rightarrow \mathrm{n}=2,21$ to $30 \% \rightarrow \mathrm{n}=3,31$ to $40 \% \rightarrow$ $\mathrm{n}=4,41$ to $50 \% \rightarrow \mathrm{n}=5,51$ to $60 \% \rightarrow \mathrm{n}=6,61$ to $70 \% \rightarrow \mathrm{n}=7,71$ to $80 \% \rightarrow \mathrm{n}=8,81$ to $90 \% \rightarrow \mathrm{n}=9$, and $>90 \% \rightarrow \mathrm{n}=10$. Then, the intensity of the staining, which varied from low to high, was estimated in a range from 1 to $3(+=1,+$ to $++=1.5,++=2,++$ to $+++=$ 2.5 , and $+++=3)$ and was added to the grading scores to obtain the final protein expression grade in each cow.

${ }^{2}$ The subcellular localization patterns of ABCG1 in MEC of lactating ( $\mathrm{d}-77$ before parturition; $\mathrm{d} 42$ after parturition) and nonlactating dairy cows (d -16, dry period) were evaluated using immunohistochemically stained section, as shown in Figure 1. a = staining of apical cytoplasm, $\mathrm{b}=$ staining of basal cytoplasm, $\mathrm{e}=$ staining of entire cytoplasm, $\mathrm{m}=$ membrane staining.

\section{RESULTS}

\section{Semiquantitative Evaluation of ABCA1 and $A B C G 1$ Proteins in MEC at Different Stages of the Pregnancy-Lactation Cycle}

In all investigated stages, ABCA1 staining was predominantly identified in alveolar MEC (Figure 1A and Table 1), although additional staining in other cell types such as endothelial cells of blood vessels, mesenchymal stromal cells, and adipocytes was found. The ABCA1 staining of MEC was very high with respect to both the fraction of positive cells and staining intensity on $\mathrm{d}-77$ and $\mathrm{d}-16$ (Table 1 ). All MEC stained positive for ABCA1 with moderate to high intensity (Figure 1A). On d 42 a smaller fraction of MEC was positive for ABCA1, and the staining intensity was lower as compared with that on $\mathrm{d}-77$ and $\mathrm{d}-16$ (Table 1 ). The protein expression grade of ABCA1 did not differ between $\mathrm{d}-77$ and $\mathrm{d}-16(P>0.05$, Table 2$)$, but was higher in these stages than on d $42(P<0.05$, Table 2).

In contrast to that with ABCA1, ABCG1 staining of MEC showed a pronounced inter-individual variability between the animals at various stages of $\mathrm{MG}$ development (Tables 2 and 3). The ABCG1 staining was present mainly in alveolar epithelial cells and infrequently in mesenchymal stromal cells (Figure 1B and Table 3). In general, at all stages, the majority of MEC were positive for ABCG1 in more than $50 \%$ of the cases (Table 3). Staining intensity mirrored the fraction of positive cells (i.e., it was greater when a large number of MEC stained for ABCG1 and lower with a lower percentage of ABCG1-positive MEC; Table 3). No statistical differences $(P>0.05)$ were found in ABCG1 protein expression between the 3 investigated stages (Table 2).

\section{Subcellular Localization of ABCA1 and ABCG1 in MG Tissues}

The subcellular distribution of ABCA1 and ABCG1 for each individual animal and stage of the pregnancylactation cycle is summarized in Tables 1 and 3. Figure 2 depicts the subcellular distribution of these proteins detected by immunofluorescence in the same animal analyzed at different functional stages of the MG.

Staining with ABCA1 was present in the cytoplasm of MEC, either diffusely in the entire cytosol or with accentuation in the basal or apical cytosolic compartments (Figures 1A and 2; Table 1). The subcellular distribution of ABCA1 changed throughout the pregnancy-lactation cycle. On d -77 , ABCA1 was present 

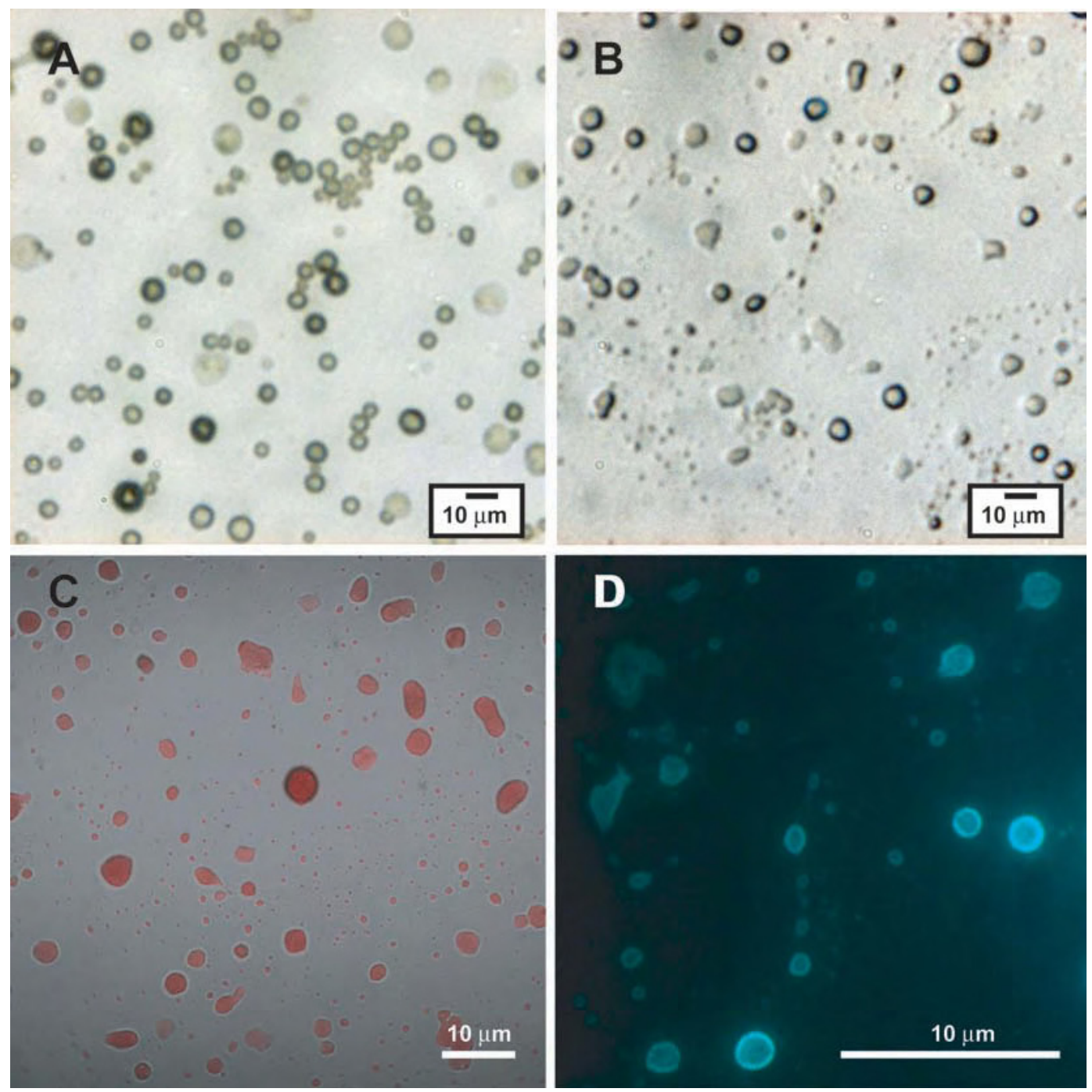

Figure 3. Characterization and identification of paraformaldehyde-fixed bovine milk fat globules (MFG) isolated from mature milk. Panel A shows the morphology of MFG $4 \mathrm{~h}$ after fixation, whereas panel B illustrates the morphology of MFG dried overnight and washed with PBS before visualization under bright field microscopy. Bright field images were recorded using a $40 \times$ objective with phase contrast. Panel C shows the confocal laser scanning overlay of fluorescence of the core triglycerides in MFG stained by oil red O dye (ORO, red). Panel D demonstrates positive staining of cholesterol in the MFG membrane by filipin (blue). Images were recorded on a fluorescence microscope using a $100 \times$ objective and Hoechst filter. Color version available in the online PDF.

diffusely in the cytoplasm with frequent basal and apical accentuation (6 of 7 animals, i.e., $85.7 \%$ of cases; Table 1; Figures 1A and 2). On d -16, mainly diffuse cytoplasmic staining with rare apical accentuation (4 of 5 animals, i.e., $80 \%$ of cases) was observed (Figures $1 \mathrm{~A}$ and 2; Table 1). In contrast, on d 42, ABCA1 was present diffusely in the cytoplasm with mostly basal accentuation ( 5 of 6 animals, i.e., $83.3 \%$ of cases). Interestingly, ABCA1 staining disappeared in all animals in the basal cytoplasmic aspect on $\mathrm{d}-16$ and in the apical cytoplasmic aspect on d 42 . In some animals (2 of 6 , i.e., $33.3 \%$ of animals), ABCA1 was identified in intraluminal secretions on $\mathrm{d}-16$ (data not shown).
The ABCG1 showed a cytoplasmic staining pattern similar to that of ABCA1. In addition, staining of the apical cell membrane was more frequently noted (Figure 1B). The subcellular distribution of ABCG1 did not change as prominently during the lactation-pregnancy cycle compared with that of ABCA1 (Table 3; Figure 1B). Staining of the cell membrane was more frequently observed on $\mathrm{d}-77$ ( 5 of 7 animals, i.e., $71.4 \%$ of cases) and $d-16$ (3 of 5 animals, i.e., $60 \%$ of cases) than on d 42 ( 1 of 6 animals, i.e., $16.7 \%$ of cases), but the pattern of cytoplasmic staining did not differ between the various stages of the pregnancy-lactation cycle (Table $3)$. Furthermore, on d -16 , ABCG1 was identified in 
intraluminal secretions in several animals (4 of 6 ; Figure 2).

\section{Characterization of MFG}

Most of the MFG showed a round intact morphology and visually unaltered structure after fixation with PFA (Figure 3A), whereas a few of them had a partly wrinkled and fused appearance (Figure $3 \mathrm{~A}$ and $\mathrm{B}$ ). The size of morphologically intact MFG varied from approximately 0.1 to $10 \mu \mathrm{m}$. As expected, triglycerides contained in the core of the MFG were present, as demonstrated by staining with ORO dye (Figure 3C, red). The morphology of attached MFG was not altered after staining with ORO (Figure 3C). Membrane cholesterol staining of the MFG showed a ring-like fluorescence pattern corresponding to cholesterol molecules present in the outer MFGM and the inner monolayer (Figure $3 \mathrm{D})$.

\section{Localization of Cholesterol Transporters in MFG}

The ABCA1 and ABCG1 proteins were localized on the membrane of permeabilized (Triton X-100-treated) and unpermeabilized (PBS-treated) MFG (Figure 4). In both cases, the morphology of MFG remained intact (Figure 4). Unpermeabilized MFG were mostly round, whereas permeabilized MFG were wrinkled and partly fused with other neighboring MFG (Figure 4A and B). Membranes of MFG in which ABCA1 or ABCG1 were labeled by specific antibodies were partly detached (green, Figure 4A, C, and D). The population of MFG staining positive for ABCA1 or ABCG1 was relatively low (Figure 4A, C and D). The MFG without ORO counterstaining showed no apparent differences in antibody staining pattern and intensity compared with those treated with ORO (Figure 4A and D).

\section{DISCUSSION}

The present study demonstrated temporal changes in ABCA1 and ABCG1 protein expression and subcellular localization in bovine mammary tissues from lactating and nonlactating dairy cows, and the presence of these proteins in MFG from mature bovine milk.

The immunofluorescence technique was employed because it was expected to allow a more sensitive detection and visualization of apical and basal localization patterns of ABCA1 and ABCG1 than does immunohistochemistry, and the identification of these proteins in MFG. For technical reasons, it was not possible to use the milk sampled from biopsied animals for MFG analysis. Nevertheless, the pooled milk samples obtained from other dairy cows allowed an initial assessment of
ABCA1 and ABCG1 expression in MFG. Although it is assumed that findings are applicable to the lactating biopsied animals, only careful interpretations are possible at this stage.

The physiological significance of the presence of ABCA1 and ABCG1 in MFG and mammary tissue may be extrapolated from the well-established role of these proteins in the regulation of cholesterol efflux in peripheral cells (Oram and Lawn, 2001; Kobayashi et al., 2006). Accordingly, ABCA1 and ABCG1 could be involved in cholesterol exchange between MEC and adjacent cells (e.g., mammary adipocytes or stromal cells within the MG). The involvement of membrane transporters in cholesterol efflux is largely associated with trafficking and localization between intracellular compartments and the plasma membrane (Santamarina-Fojo et al., 2001; Schmitz et al., 2001). In the present study, the subcellular localization of ABCA1 and ABCG1 was not stable, but rather changed in nonlactating and lactating MG. The transporters ABCA1 and ABCG1 were present diffusely in the cytoplasm, with frequent basal and apical accentuation during the end of the previous lactation $(\mathrm{d}-77)$, whereas on $\mathrm{d}-16$, proteins rarely exhibited apical accentuation. On d 42, ABCA1 and ABCG1 showed mostly basal accentuation. Thus, the localization patterns seem to depend on the functional stage of the MG. The observed subcellular localization of ABCA1 and ABCG1 indicated the presence of these major cholesterol transporters in the plasma membrane and cytoplasmic intracellular compartments of MEC. The presence of ABCA1 toward the basal cytoplasm suggests that the basolateral efflux of cholesterol onto stromal cholesterol acceptors (such as apolipoproteins or lipoproteins) is likely. In vitro studies performed in human embryonic kidney (HEK) and HeLa cells reported the presence of ABCA1 in endosomal vesicles that shuttle between the endoplasmic reticulum and the plasma membrane (Neufeld et al., 2001), where the protein is thought to be recycled back through retroendocytosis (Azuma et al., 2009).

Milk cholesterol release occurs at the luminal MEC plasma membrane (Easter, 1971; McManaman and Neville, 2003). In order to convincingly demonstrate the possible involvement of ABCA1 and ABCG1 in this process, their presence was examined in the MFG because the outer lipid bilayer surrounding MFG originates from the apical part of MEC (McManaman and Neville, 2003). Immunofluorescence data confirmed the presence of ABCA1 and ABCG1 in MFG, consistent with an apical localization and a role in milk cholesterol transfer (Easter, 1971; McManaman and Neville, 2003). The finding that only a fraction of MFG was stained for ABCA1 and ABCG1 could be because the milk used for isolation of MFG was a mixture repre- 


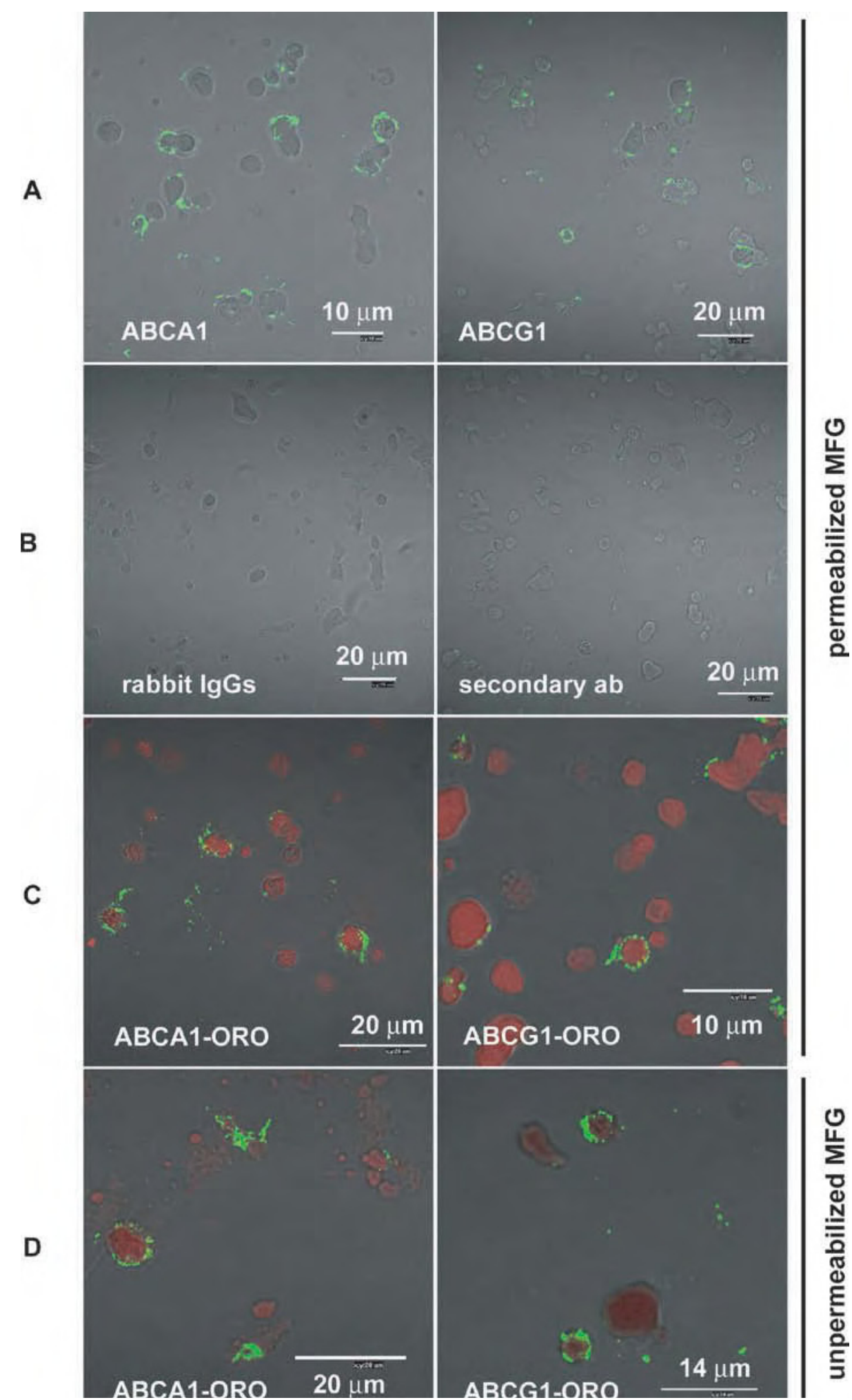

Figure 4. Immunofluorescence staining of the ATP-binding cassette transporters A1 (ABCA1) and G1 (ABCG1) in bovine milk fat globules (MFG) isolated from mature milk. Permeabilized (panels A, B, C) and unpermeabilized (panel D) MFG were stained with anti-ABCA1 or antiABCG1 antibodies and detected by Alexa Fluor 488 fluorophore conjugated secondary antibodies (green). The MFG in panels C and D were counterstained with oil red $\mathrm{O}$ dye (ORO, red). Negative controls are shown in panel B. For the detection of ABCA1 and ABCG1 by laser scanning confocal microscopy, a $100 \times$ objective was used. The arrows indicate positive staining for ABCA1 (left panels) or ABCG1 (right panels) on the membrane of MFG. Color version available in the online PDF. 
senting a spectrum of lactational stages from wk 2 to 40. The present as well as previous studies (Mani et al., 2009, 2010) revealed that the localization of ABCA1 in MEC seemed to change between early lactation (d 42 , predominantly basolateral localization) and later lactational stages ( $\mathrm{d}-77$ and 88 , often apical accentuation). On the other hand, the apparent lack of ABCA1 accumulation at the apical membrane might be due to a rapid flux of membrane components into the MFGM. Therefore, future studies should address the question of whether the percentage of positively stained MFG is indeed associated with the lactational stage and should determine the degree of variation between individual animals.

Within the scope of this study, it was not possible to determine whether ABCA1 and ABCG1 mediate the cholesterol transfer by acting as self-operating transporters or as components of the MFG secretion pathway. Intriguingly, it was reported that key acceptors of cholesterol efflux by ABCA1, such as apo A1 and apo E (Ikonen, 2008), are present in MFG prepared from colostrum and mature milk (Mather and Keenan, 1998; Fong et al., 2007). The physiological significance of ABCA1 and ABCG1 expression in the MFG is not yet clear. As it is questionable whether ABCA1 and ABCG1 dispose of sufficient ATP to elicit physiological activities in the MFG or to initiate likely exchanges with MEC, it is tempting to speculate that the presence of ABCA1 and ABCG1 in MFG indicates losses of these proteins in MEC (i.e., they are rapidly turned over with the formation of MFG sustaining the milk secretion). If this is confirmed, it may a priori imply that in contrast to the situation in other cells (Neufeld et al., 2001; Azuma et al., 2009), ABCA1 and ABCG1 are not (or only to a lesser extent) recycled back from the apical side of the MEC plasma membrane when milk secretion is ongoing. Whether these proteins act differently at the basal MEC plasma membrane during sustained milk secretion and at both sides of MEC plasma membrane when milk secretion ceases, requires further investigation.

Unlike that of ABCA1, the protein expression and localization of ABCG1 in biopsied animals was the same regardless of the functional stage of the $\mathrm{MG}$, implying a differential role for ABCA1 and ABCG1 in MG physiology. It is known that ABCA1 is involved in the removal of excess cholesterol from peripheral tissues by reverse cholesterol transport (Attie et al., 2001; Cavelier et al., 2006; Oram and Vaughan, 2006), a fundamental mechanism that might also be important in MEC. Previous studies (Mani et al., 2009) demonstrated a significant decrease in the levels of circulating cholesterol in nonlactating $(\mathrm{d}-16)$ compared with those in lactating (e.g., $\mathrm{d}$ 42) cows. Concomitantly, ABCA1 protein expression in
MEC was increased on $\mathrm{d}-16$ as compared with expression on $\mathrm{d} 42$. These results indicate that high ABCA1 expression coincides with the period of MG remodeling rather than with acute milk secretion. A potential link between ABCA1 and MG remodeling might be derived from recent studies demonstrating an interaction between ABCA1 and janus kinase (Vaughan et al., 2009) with downstream effects on signaling proteins such as the "signal transducer activator of transcription." The isoform 3 of this family, which is potentially targeted in an ABCA1-dependent manner in MEC, has been identified as a key regulator of involution-associated epithelial cell death and mammary tissue remodeling (Humphreys et al., 2002; Zhao et al., 2004).

\section{CONCLUSIONS}

In summary, ABCA1 and ABCG1 were detected in MFG, providing support for the physiological importance of these major cholesterol transporters in milk cholesterol release. Furthermore, the localization of ABCA1 and ABCG1 at the basal and apical cytoplasm suggests that these proteins may share a common function in the regulation of cholesterol homeostasis in the MG or exchanges between MEC and the surroundings (including the alveolar lumen). The results of the present study will serve as a basis for discussing potential regulatory mechanisms and functions of $\mathrm{ABCA} 1$ and ABCG1 in the MG. Yet, additional MFG investigations in relation to changing milk composition over the course of lactation, as well as in vitro lipid transport assays using cultured MEC, are needed to supplement the present findings.

\section{ACKNOWLEDGMENTS}

This project was supported by grants from the Novartis Foundation, the Wolfermann-Nägeli Stiftung and the SwissLife Jubiläumsstiftung. We thank C. Wotzkow (Institute of Biochemistry and Molecular Medicine, University of Bern, Switzerland) for his valuable help with immunohistochemistry.

\section{REFERENCES}

Attie, A. D., J. P. Kastelein, and M. R. Hayden. 2001. Pivotal role of ABCA1 in reverse cholesterol transport influencing HDL levels and susceptibility to atherosclerosis. J. Lipid Res. 42:1717-1726.

Azuma, Y., M. Takada, H. W. Shin, N. Kioka, K. Nakayama, and K. Ueda. 2009. Retroendocytosis pathway of ABCA1/apoA-I contributes to HDL formation. Genes Cells 14:191-204.

Bitman, J., D. L. Wood, and A. V. Capuco. 1992. Changes in the lipid composition of the secretions of the bovine mammary gland during the dry period. J. Dairy Sci. 75:435-442.

Cavelier, C., I. Lorenzi, L. Rohrer, and A. von Eckardstein. 2006. Lipid efflux by the ATP-binding cassette transporters ABCA1 and ABCG1. Biochim. Biophys. Acta 1761:655-666. 
Easter, D. J. 1971. The turnover time of dietary cholesterol in the lactating rat. Lipids 6:645-648.

Fong, B. Y., C. S. Norris, and A. K. H. MacGibbon. 2007. Protein and lipid composition of bovine milk-fat-globule membrane. Int. Dairy J. $17: 275-288$.

Fortunato, D., M. G. Giuffrida, M. Cavaletto, L. P. Garoffo, G. Dellavalle, L. Napolitano, C. Giunta, C. Fabris, E. Bertino, A. Coscia, and A. Conti. 2003. Structural proteome of human colostral fat globule membrane proteins. Proteomics 3:897-905.

Humphreys, R. C., B. Bierie, L. Zhao, R. Raz, D. Levy, and L. Hennighausen. 2002. Deletion of Stat3 blocks mammary gland involution and extends functional competence of the secretory epithelium in the absence of lactogenic stimuli. Endocrinology 143:3641-3650.

Ikonen, E. 2008. Cellular cholesterol trafficking and compartmentalization. Nat. Rev. Mol. Cell Biol. 9:125-138.

Ioannou, Y. A. 2001. Multidrug permeases and subcellular cholesterol transport. Nat. Rev. Mol. Cell Biol. 2:657-668.

Kobayashi, A., Y. Takanezawa, T. Hirata, Y. Shimizu, K. Misasa, N. Kioka, H. Arai, K. Ueda, and M. Matsuo. 2006. Efflux of sphingomyelin, cholesterol, and phosphatidylcholine by ABCG1. J. Lipid Res. 47:1791-1802.

Mani, O., M. Körner, M. T. Sorensen, K. Sejrsen, C. Wotzkow, C. E. Ontsouka, R. R. Friis, R. M. Bruckmaier, and C. Albrecht. 2010. Expression, localization and functional model of cholesterol transporters in lactating and nonlactating mammary tissues of murine, bovine, and human origin. Am. J. Physiol. Regul. Integr. Comp. Physiol. 299:R642-R654.

Mani, O., M. T. Sorensen, K. Sejrsen, R. M. Bruckmaier, and C. Albrecht. 2009. Differential expression and localization of lipid transporters in the bovine mammary gland during the pregnancylactation cycle. J. Dairy Sci. 92:3744-3756.

Mather, I. H., and T. W. Keenan. 1998. Origin and secretion of milk lipids. J. Mammary Gland Biol. Neoplasia 3:259-273.

McManaman, J. L., and M. C. Neville. 2003. Mammary physiology and milk secretion. Adv. Drug Deliv. Rev. 55:629-641.

Mohsin, S. K., H. Weiss, T. Havighurst, G. M. Clark, M. Berardo, L. D. Roanh, T. V. To, Z Qian, R. R. Love, and D. C. Allred. 2004. Progesterone receptor by immunohistochemistry and clinical outcome in breast cancer: A validation study. Mod. Pathol. $17: 1545-1554$

Neufeld, E. B., A. T. Remaley, S. J. Demosky, J. A. Stonik, A. M. Cooney, M. Comly, N. K. Dwyer, M. Zhang, J. Blanchette-Mackie, S. Santamarina-Fojo, and H. B. Brewer Jr. 2001. Cellular localiza- tion and trafficking of the human ABCA1 transporter. J. Biol. Chem. 276:27584-27590.

Oram, J. F., and R. M. Lawn. 2001. ABCA1. The gatekeeper for eliminating excess tissue cholesterol. J. Lipid Res. 42:1173-1179.

Oram, J. F., and A. M. Vaughan. 2006. ATP-binding cassette cholesterol transporters and cardiovascular disease. Circ. Res. 99:10311043.

Paterson, D. A., C. P. Reid, T. J. Anderson, and R. A. Hawkins. 1990. Assessment of oestrogen receptor content of breast carcinoma by immunohistochemical techniques on fixed and frozen tissue and by biochemical ligand binding assay. J. Clin. Pathol. 43:46-51.

Paul, V., H. H. D. Meyer, K. Leidl, S. Soumian, and C. Albrecht. 2008. A novel enzyme immunoassay specific for ABCA1 protein quantification in human tissues and cells. J. Lipid Res. 49:2259-2267.

Reinhardt, T. A., and J. D. Lippolis. 2008. Developmental changes in the milk fat globule membrane proteome during the transition from colostrum to milk. J. Dairy Sci. 91:2307-2318.

Robenek, H., O. Hofnagel, I. Buers, S. Lorkowski, M. Schnoor, M. J. Robenek, H. Heid, D. Troyer, and N. J. Severs. 2006. Butyrophilin controls milk fat globule secretion. Proc. Natl. Acad. Sci. USA 103:10385-10390.

Santamarina-Fojo, S., A. T. Remaley, E. B. Neufeld, and H. B. Brewer Jr.. 2001. Regulation and intracellular trafficking of the ABCA1 transporter. J. Lipid Res. 42:1339-1345.

Schmitz, G., T. Langmann, and S. Heimerl. 2001. Role of ABCG1 and other ABCG family members in lipid metabolism. J. Lipid Res. 42:1513-1520.

Sorensen, M. T., J. V. Nørgaard, P. K. Theil, M. Vestergaard, and K. Sejrsen. 2006. Cell turnover and activity in mammary tissue during lactation and the dry period in dairy cows. J. Dairy Sci. 89:4632-4639

Takahashi, K., Y. Kimura, K. Nagata, A. Yamamoto, M. Matsuo, and K. Ueda. 2005. ABC proteins: Key molecules for lipid homeostasis. Med. Mol. Morphol. 38:2-12.

Vaughan, A. M., C. Tang, and J. F. Oram. 2009. ABCA1 mutants reveal an interdependency between lipid export function, apoAI binding activity, and Janus kinase 2 activation. J. Lipid Res. 50:285-292.

Zhao, L., S. Hart, J. Cheng, J. J. Melenhorst, B. Bierie, M. Ernst, C. Stewart, F. Schaper, P. C. Heinrich, A. Ullrich, G. W. Robinson, and L. Hennighausen. 2004. Mammary gland remodeling depends on gp130 signaling through Stat3 and MAPK. J. Biol. Chem. 279:44093-44100. 